\title{
VERNALIZAÇÃO AFETA O FILOCRONO EM LÍRIO ${ }^{(1)}$
}

\author{
MARIÂNGELA SCHUH ${ }^{(2)}$; NEREU AUGUSTO STRECK ${ }^{(3)}$; CLAUDIA NARDI $^{(3)}$; \\ GALILEO ADELI BURIOL ${ }^{(4)}$; ROGÉRIO ANTÔNIO BELLÉ ${ }^{(3)}$; AURI BRACKMANN ${ }^{(4)}$
}

\begin{abstract}
RESUMO
O experimento foi desenvolvido com o objetivo de estudar o efeito da vernalização sobre o filocrono (intervalo de tempo entre o aparecimento de duas folhas sucessivas) em lírio (Lilium longiflorum Thunb.), cv. Snow Queen, no campo experimental do Departamento de Fitotecnia da Universidade Federal de Santa Maria (UFSM), Santa Maria (RS). Os tratamentos constituíram-se de bulbos submetidos a $-0,5{ }^{\circ} \mathrm{C} ; 4$ ${ }^{\circ} \mathrm{C}$ e $10{ }^{\circ} \mathrm{C}$ com períodos de vernalização dos bulbos de 2, 4, 6 e 8 semanas. O tratamento testemunha correspondeu a bulbos não vernalizados. Após o tratamento de vernalização, os bulbos foram plantados no interior de uma estufa plástica em 5 de dezembro de 2002 e observado o desenvolvimento das plantas. O filocrono foi estimado pelo inverso do coeficiente angular da regressão linear entre o número de folhas e o acúmulo térmico acima de $1{ }^{\circ} \mathrm{C}$, a partir da emergência das plantas. Os resultados evidenciaram que o estado de vernalização da planta afeta o filocrono em lírio, e plantas não vernalizadas ou com pouca vernalização têm um filocrono mais elevado do que plantas completamente vernalizadas. Quando o tratamento de vernalização é maior que 30 dias efetivos de vernalização, o valor do filocrono foi em torno de $25{ }^{\circ} \mathrm{C}$ dia / folha, valor que pode ser utilizado como referência quando os bulbos são vernalizados e utilizados em plantios comerciais.
\end{abstract}

Palavras-chave: Lilium longiflorum Thunb., filocrono, vernalização, emissão de folhas, acúmulo térmico.

\section{ABSTRACT \\ VERNALIZATION AFFECTS THE PHYLLOCHRON IN LILY}

An experiment was carried out to verify the effect of vernalization period on the phyllochron (time interval between the appearance of two successive leaves) in lily (Lilium longiflorum Thunb), cv. 'Snow Queen'. The trial was conducted at Santa Maria, RS, Brazil. Treatments were bulbs stored at vernalizing temperatures of $-0.5,4$, and $10^{\circ} \mathrm{C}$ during periods of $2,4,6$ and 8 weeks. Control treatment was unvernalized bulbs. Bulbs were planted inside a plastic greenhouse on December 05, 2002, and crop development was evaluated throughout the growing season. Phyllochron for each treatment was estimated as the inverse of the slope of the linear regression relating leaf number and accumulated thermal time above $1^{\circ} \mathrm{C}$ after emergence. Results showed that the phyllochron is affected by vernalization. Unvernalized plants and plants with low vernalization treatments had greater phyllochron values compared to completely vernalized plants. Plants vernalized with 30 or higher effective vernalization days had a phyllochron value around $25{ }^{\circ} \mathrm{C}$ days/leaf. This phyllochron can be used as a reference when vernalized bulbs are used in commercial croppings.

Key words: Lilium longiflorum Thunb., phyllochron, vernalization, leaf unfolding, thermal time.

( $\left.{ }^{1}\right)$ Recebido para publicação em 9 de dezembro de 2003 e aceito em 20 de outubro de 2004 .

( ${ }^{2}$ ) Aluna do Curso de Graduação em Agronomia, Centro de Ciências Rurais (CCR), Universidade Federal de Santa Maria (UFSM), 97105-900 Santa Maria (RS). Bolsista FIPE/UFSM.

$\left({ }^{3}\right)$ Departamento de Fitotecnia/CCR/UFSM. E-mail: nstreck1@smail.ufsm.br.

$\left({ }^{4}\right)$ Bolsista do CNPq. 


\section{INTRODUÇÃO}

O lírio pertence ao grupo das plantas bulbosas e é uma cultura constituída por grande número de espécies, híbridos e cultivares comercialmente disponíveis. O Lilium longiflorum, mais conhecido como "lírio branco", é muito usado para a produção de flores de corte.

O lírio requer exposição dos bulbos a baixas temperaturas do ar para entrar na fase reprodutiva (RoH E Wilkins, 1977a; RoH, 1985); esse processo denomina-se vernalização. Na planta de lírio oriunda de bulbos submetidos a temperaturas vernalizantes, observam-se emergência mais rápida e uniforme e diminuição do ciclo de desenvolvimento, com florescimento mais precoce comparada com outra oriunda de bulbos não vernalizados. A diminuição no tempo para florescer é causada por decréscimo no número de primórdios folhares, ou seja, no número final de folhas (RoH e WiLKINS, 1977b,c; RoH, 1985). Segundo Mота (1977), a vernalização constitui-se em um processo de acumulação de baixas temperaturas por parte da planta, desde o estágio de semente germinada até o momento da formação do talo.

Vários fatores ecológicos influenciam o crescimento e o desenvolvimento da planta de lírio. Entre esses fatores, a temperatura do ar afeta diferentes processos na planta incluindo a taxa de emissão de folhas (KARLSSON et al., 1988; Lieth e CARPENTER, 1990). A medida ou estimativa da emissão de folhas é importante, pois, integrando-se a taxa de emissão de folhas tem-se o número de folhas acumulado (NF) na haste, que é uma excelente medida da idade fisiológica da planta. O NF também está associado à área folhar da planta, responsável por interceptar a radiação solar pelo dossel vegetal para ser usada na fotossíntese.

A emissão de folhas pode ser estimada utilizando-se o conceito do filocrono. Wilhelm e MCMASTER (1995) definem o filocrono como o intervalo entre estágios similares de desenvolvimento de folhas sobre o ápice. KLEPPER et al. (1982) definem o filocrono como o intervalo de tempo entre a emissão de duas folhas sucessivas sobre o primórdio vegetativo de gramíneas. Portanto, pode-se definir o filocrono como o intervalo de tempo entre o aparecimento de folhas sucessivas. Uma unidade de tempo freqüentemente utilizada para representar tempo fisiológico em plantas é a soma térmica ou soma de calor $\left({ }^{\circ} \mathrm{C}\right.$ dia $)$. O filocrono, neste caso, é o acúmulo térmico necessário para o aparecimento de uma folha $\left({ }^{\circ} \mathrm{C}\right.$ dia / folha).

Embora exista unanimidade de que a vernalização afeta o número final de folhas na haste principal de gramíneas, como o trigo de inverno
(Cutforth et al., 1992; Streck et al., 2003 a,c) e em lírio (Roh e Wilkins, 1977b,c; RoH, 1985), o efeito da vernalização sobre a taxa de emissão folhar é um assunto ainda em debate. Por exemplo, em trigo existem evidências de que plantas não vernalizadas apresentam menor taxa de emissão de folhas, ou seja, maior filocrono (CUTFORTH et al., 1992; CHUN, 1993; RAwsON et al., 1998). Há estudos, também, que não mostraram essa tendência e sim que a taxa de emissão de folhas de plantas de trigo não vernalizadas é similar à taxa de emissão de folhas de plantas vernalizadas (CAO e Moss, 1991; MOSAAD et al., 1995; StRECK et al., 2003b). Não foram encontrados estudos a respeito do efeito da vernalização sobre a taxa de emissão de folhas ou o filocrono em lírio, o que constituiu o incentivo para realizar o presente estudo, cujo objetivo foi estudar o efeito da vernalização sobre o filocrono em lírio.

\section{MATERIAL E MÉTODOS}

O experimento foi desenvolvido no Campo Experimental do Departamento de Fitotecnia da Universidade Federal de Santa Maria, Santa Maria, RS (latitude: $29^{\circ} 41^{\prime} \mathrm{S}$, longitude: $53^{\circ} 41^{\prime} \mathrm{W}$ e altitude: $95 \mathrm{~m}$ ). Foi utilizada a cultivar Snow Queen, de flor branca, considerada de boa adaptabilidade e produtividade para as condições ambientais brasileiras. Os bulbos foram acondicionados em fibra de coco e colocados em sacos de polietileno, e submetidos a diferentes temperaturas e tempos de vernalização (Tabela 1) em câmaras refrigeradoras instaladas no núcleo de PósColheita do Departamento de Fitotecnia da Universidade Federal de Santa Maria, de modo que todos os tratamentos foram completados em 5 de dezembro de 2002. Os bulbos do tratamento-controle (sem vernalização) ficaram em uma sala com temperatura sempre maior que $25^{\circ} \mathrm{C}$.

O tempo de exposição a diferentes temperaturas de vernalização pode ser medido por dias efetivos de vernalização (DV). Um dia efetivo de vernalização (DV) é obtido quando a planta ou o bulbo são expostos à temperatura ótima de vernalização, durante 24 horas (STRECK, 2002b). Sempre que a temperatura for inferior ou superior à temperatura ótima de vernalização, somente uma fração de um DV é acumulada pela planta durante um dia do calendário oficial. Portanto, a representação do estado de vernalização da planta através de DV é apropriada, pois no cálculo já estão considerados a temperatura e o tempo de exposição a temperaturas vernalizantes, o que é realístico do ponto de vista biológico (StRECK et al., 2003a). 
Tabela 1. Tratamentos e dias efetivos de vernalização (DV) correspondentes às temperaturas de vernalização e às durações dos períodos de armazenamento para a cultura do lírio (Lilium longiflorum Thunb.) cv. Snow Queen utilizados neste estudo. Santa Maria (RS), 2003

\begin{tabular}{|c|c|c|}
\hline \multicolumn{2}{|c|}{ Tratamentos } & \multirow{2}{*}{$\begin{array}{c}\text { Dias efetivos de vernalização } \\
\text { (DV) }\end{array}$} \\
\hline Temperatura & Duração (semanas) & \\
\hline $10{ }^{\circ} \mathrm{C}$ & 2 & 12,04 \\
\hline $10{ }^{\circ} \mathrm{C}$ & 4 & 24,08 \\
\hline $10{ }^{\circ} \mathrm{C}$ & 6 & 36,12 \\
\hline $10{ }^{\circ} \mathrm{C}$ & 8 & 46,44 \\
\hline $4{ }^{\circ} \mathrm{C}$ & 2 & 13,89 \\
\hline $4{ }^{\circ} \mathrm{C}$ & 4 & 27,78 \\
\hline $4{ }^{\circ} \mathrm{C}$ & 6 & 41,66 \\
\hline $4{ }^{\circ} \mathrm{C}$ & 8 & 53,57 \\
\hline$-0,5^{\circ} \mathrm{C}$ & 2 & 8,12 \\
\hline$-0,5^{\circ} \mathrm{C}$ & 4 & 16,24 \\
\hline$-0,5^{\circ} \mathrm{C}$ & 6 & 24,36 \\
\hline$-0,5^{\circ} \mathrm{C}$ & 8 & 31,32 \\
\hline Sem vernalizar & - & 0 \\
\hline
\end{tabular}

Para o cálculo dos dias efetivos de vernalização para cada tratamento em que os bulbos de lírio foram submetidos nas câmaras refrigeradoras (Tabela 1), foi utilizado o seguinte método. A resposta (variação de 0 a 1) da vernalização do Lilium longiflorum à temperatura pode ser denominada taxa diária de vernalização [fvn(T), dia ${ }^{-1}$ ] (STRECK, 2002b, STRECK et al., 2003a), e foi descrita por uma função beta. A função beta tem três coeficientes que, no presente estudo, são as temperaturas cardeais de vernalização (STRECK, 2002a), sendo definida como:

$\operatorname{fvn}(\mathrm{T})=\left[2(\mathrm{~T}-\mathrm{Tmin})^{\mathrm{a}}(\text { Tót-Tmin })^{\mathrm{a}}-(\mathrm{T}-\mathrm{Tmin})^{2 \mathrm{a}}\right] /($ Tót $\operatorname{Tmin})^{2 a}$

$\mathrm{a}=\ln 2 / \ln [($ Tmax-Tmin) $/($ Tót-Tmin $)]$

em que: Tmin = temperatura mínima; Tót $=$ temperatura ótima; Tmax = temperatura máxima de vernalização e $\mathrm{T}=$ temperatura de armazenamento dos bulbos durante a vernalização $\left(-0,5 ; 4\right.$ e $\left.10^{\circ} \mathrm{C}\right)$. Considerou-se que as temperaturas cardeais de vernalização do lírio são $-1,5^{\circ} \mathrm{C}$ (MCRAE, 1998; LeE e RoH, 2001), $5{ }^{\circ} \mathrm{C}$ e $21{ }^{\circ} \mathrm{C}$ (Streck, 2002b). Com essas pressuposições, a resposta da função beta usada no cálculo de fvn(T) (Equações 1 e 2) é apresentada na Figura 1.
Os dias efetivos de vernalização (DV) foram calculados pela equação (STRECK, 2002b, StRECK ET al., 2003a):

$$
\mathrm{DV}=\mathrm{Sfvn}(\mathrm{T})
$$

em que: $f v n(T)$ - foi definida anteriormente e calculada por meio das equações (1) e (2) com as temperaturas cardeais para a vernalização do lírio que são $-1,5^{\circ} \mathrm{C}, 5^{\circ} \mathrm{C}$ e $21^{\circ} \mathrm{C}$.

O plantio dos bulbos de lírio foi realizado em 5 de dezembro de 2002, em estufa plástica com estrutura metálica, com área de $180 \mathrm{~m}^{2}$ e ventilação natural. No interior da estufa plástica, o desenho experimental foi o de parcelas subdivididas em blocos ao acaso, com três repetições. Os tratamentos foram temperatura de vernalização (parcela principal) e tempo de vernalização (subparcela) (Tabela 1). Canteiros de $1 \mathrm{~m} \times 3 \mathrm{~m}$ foram distribuídos no sentido do comprimento da estufa e orientados na direção norte-sul. $\mathrm{O}$ espaçamento usado entre as plantas foi $15 \mathrm{~cm} \times 15 \mathrm{~cm}$. A subparcela constou de três fileiras com quatro plantas/fileira, totalizando doze plantas. Os parâmetros de desenvolvimento vegetal de interesse neste estudo foram medidos em duas plantas na fileira central de cada subparcela (parcela útil), tendo-se as demais plantas como bordadura e não utilizadas na coleta de dados. 


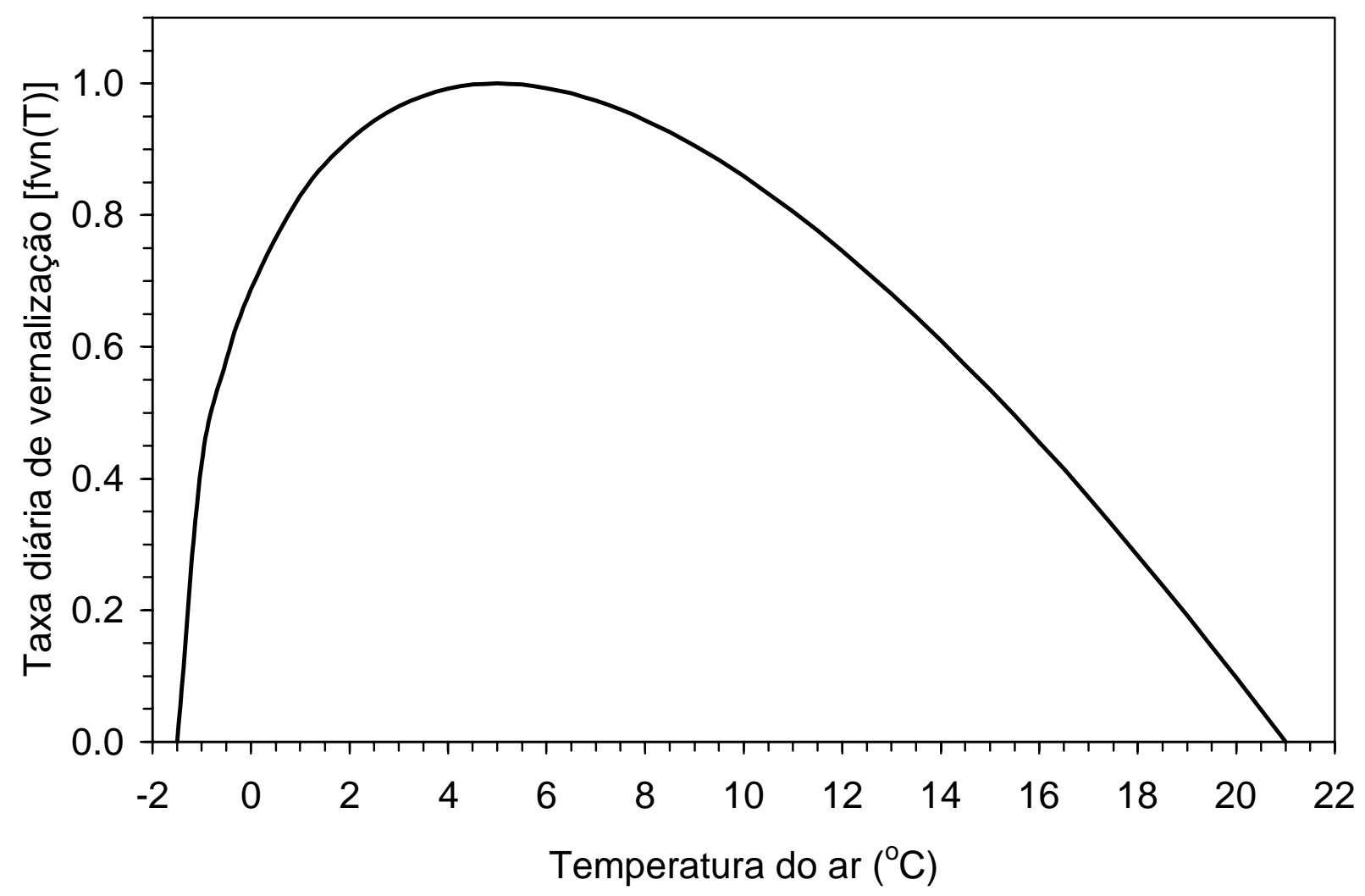

Figura 1. Taxa diária de vernalização [fvn(T)] em lírio (Lilium longiflorum Thunb.) cv. Snow Queen na faixa de temperaturas cardeais de sua vernalização.

A temperatura do ar no interior da estufa foi registrada por um termo-higrógrafo a $1,5 \mathrm{~m}$ do solo, instalado dentro de um abrigo meteorológico pintado de branco na parte central dentro da estufa. Dos termohigrogramas foram retirados os valores de temperatura mínima e máxima diárias.

As folhas na haste das plantas de lírio foram contadas nas duas plantas úteis/subparcela quando estavam desprendidas da parte apical da roseta (KARLSSON et al., 1988). Contou-se o número de folhas duas vezes por semana, desde o aparecimento da primeira folha até o desenvolvimento do botão floral, quando cessou a emissão de folhas.

A soma térmica diária (STd) no interior da estufa foi calculada por:

$$
\mathrm{STd}=\mathrm{Tm}-\mathrm{Tb}
$$

em que: $\mathrm{Tm}=$ temperatura média diária do ar no interior da estufa, calculada pela média aritmética das temperaturas mínima e máxima do $\mathrm{ar} ; \mathrm{Tb}=$ temperatura base para aparecimento de folhas em lírio, considerada como $1^{\circ} \mathrm{C}$ (KARLSSON et al., 1988).
A soma térmica acumulada no interior da estufa (STa) foi calculada por:

$$
\mathrm{STa}=\mathrm{a} S \mathrm{~T}
$$

A data considerada como primeiro dia de soma térmica acumulada (STa) foi aquele em que ocorreu $50 \%$ de emergência das plantas do respectivo tratamento; o último dia foi 7 de abril de 2003, pois após essa data a temperatura mínima no interior da estufa atingiu $10^{\circ} \mathrm{C}$, valor que tem efeito vernalizante sobre a planta e, portanto, poderia interferir nos tratamentos.

Usou-se a relação número de folhas acumulada na haste (NF) e soma térmica acumulada (STa) para a estimativa do filocrono em lírio.

O filocrono foi estimado como o inverso do coeficiente angular $(b)$ da regressão linear $[N F=a+$ b(STa)] (CAO e Moss, 1991). Com os valores estimados de filocrono para cada DV, a relação entre DV e filocrono foi obtida por análise de regressão. 


\section{RESULTADOS E DISCUSSÃO}

O número total médio de folhas por planta nos tratamentos: $10{ }^{\circ} \mathrm{C}$ durante 4 semanas; $10{ }^{\circ} \mathrm{C}$ durante 6 semanas; $10{ }^{\circ} \mathrm{C}$ durante 8 semanas; $4{ }^{\circ} \mathrm{C}$ durante 6 semanas e $4{ }^{\circ} \mathrm{C}$ durante 8 semanas foi de 43,$5 ; 41,6$; 42,$5 ; 41,3$ e 39,3 folhas respectivamente. Todas as plantas desses tratamentos atingiram o estádio de botão visível até o fim do experimento, ou seja, entraram na fase reprodutiva. Já nas plantas dos tratamentos $10{ }^{\circ} \mathrm{C}$ durante 2 semanas; $4{ }^{\circ} \mathrm{C}$ durante 2 semanas; $4{ }^{\circ} \mathrm{C}$ durante 4 semanas; $-0,5^{\circ} \mathrm{C}$ durante 2 semanas; $-0,5^{\circ} \mathrm{C}$ durante 4 semanas; $-0,5^{\circ} \mathrm{C}$ durante 6 semanas; $-0,5^{\circ} \mathrm{C}$ durante 8 semanas e no tratamentotestemunha (sem vernalização), tiveram o número médio de folhas por planta em 19 de maio de 47;39,5; 49 ; 77; 67,2; 58,2; 55,8; e 17,5 folhas respectivamente; as plantas desses tratamentos permaneceram na fase vegetativa até esse dia, quando o experimento foi encerrado, não atingindo o estágio de botão visível, quando cessa a emissão de folhas (KARLSSON et al., 1988).

A relação entre o número de folhas na haste da planta (NF) e a soma térmica acumulada (STa) utilizada para a estimativa do filocrono nos diferentes tratamentos de vernalização está na Figura 2. O coeficiente de determinação $\left(R^{2}\right)$ foi 0,97 ou maior para a maioria dos tratamentos, com exceção do tratamento $10{ }^{\circ} \mathrm{C}$ durante 2 semanas, que teve um $\mathrm{R}^{2}=0,84$. Esses elevados valores de $\mathrm{R}^{2}$ comprovam que a temperatura do ar é um fator ecológico principal que governa a emissão de folhas em lírio, pois, sendo $\mathrm{R}^{2}$ elevado, a variação da variável dependente (NF) é grandemente explicada pela variação da variável independente (STa).

Esse resultado concorda com Le NARD (1994) quando afirma que o conhecimento da influência da temperatura sobre os diversos processos de crescimento e desenvolvimento constitui-se na base técnica para o controle do ciclo de desenvolvimento das plantas bulbosas. FISHER et al. (1997a,b) desenvolveram um sistema de suporte para simular em tempo real o aparecimento de folhas e a fenologia do lírio também com base na temperatura do ar dentro da estufa de produção.

O filocrono estimado para o lírio Snow Queen variou de 19,3 a $200,0{ }^{\circ} \mathrm{C}$ dia/folha, em vista do tratamento de vernalização (Figura 2). Nos tratamentos $10^{\circ} \mathrm{C}$ durante 6 semanas, $10^{\circ} \mathrm{C}$ durante 8 semanas e $4{ }^{\circ} \mathrm{C}$ durante 8 semanas verificaram-se os menores valores de filocrono $\left(22,1 ; 19,3^{\circ} \mathrm{C}\right.$ e 22,0 dia / folha). Já no tratamento-testemunha (sem vernalização) observou-se o maior valor de filocrono (200,0 ${ }^{\circ} \mathrm{C}$ dia/folha).
Na figura 3 está representado o filocrono do lírio Snow Queen em função de dias efetivos de vernalização. Os dados observados na Figura 3 são os valores de filocrono estimados para cada tratamento de vernalização apresentados na Figura 2, após os tratamentos de vernalização terem sido transformados em dias efetivos de vernalização (Tabela 1). A medida do estado de vernalização das plantas através dos dias efetivos de vernalização (DV) leva em conta o efeito da temperatura e do tempo de exposição a temperaturas vernalizantes (STRECK, 2002b; StReCK et al., 2003a), o que torna mais apropriada a representação da resposta da planta à vernalização comparada com temperaturas e semanas.

Pelos dados da figura 3, observou-se que à medida que aumentou o estado de vernalização da planta, ou seja, aumentou DV, o filocrono diminuiu de forma exponencial. Uma equação exponencial negativa representou a maior parte da variação dos dados, com um $R^{2}=0,76$. Nota-se que a diminuição do filocrono foi maior até aproximadamente 30 dias efetivos de vernalização. Acima de $30 \mathrm{DV}$, o valor do filocrono ficou em torno de $25^{\circ} \mathrm{C}$ dia / folha.

Com base nesses resultados, pode-se afirmar que uma planta de lírio com necessidades de vernalização atendidas (o que se pode chamar de planta completamente vernalizada) tem o valor do filocrono de aproximadamente $25^{\circ} \mathrm{C}$ dia/folha. Essa informação é importante do ponto de vista da modelagem do aparecimento de folhas na planta de lírio (FISHER et al., 1997a,b), pois quando o produtor planta bulbos vernalizados, pode-se usar esse valor de filocrono para estimar o aparecimento de folhas em lírio em um plantio comercial.

O principal efeito de um tratamento de vernalização dos bulbos de lírio é o encurtamento do ciclo de desenvolvimento da planta (RoH e Wilkins, 1977a; RoH, 1985). Houve diminuição do número de folhas/planta em função do aumento do estado de vernalização das plantas neste ensaio. Plantas dos tratamentos com menor vernalização $\left[8 \mathrm{DV}\left(-0,5{ }^{\circ} \mathrm{C}\right.\right.$ durante 2 semanas $), 12 \mathrm{DV}\left(10^{\circ} \mathrm{C}\right.$ durante 2 semanas $)$, 14 DV $\left(4{ }^{\circ} \mathrm{C}\right.$ durante 2 semanas $)$ e 16 DV $\left(-0,5{ }^{\circ} \mathrm{C}\right.$ durante 4 semanas)] tiveram 39 a 77 folhas/planta até o último dia do experimento, quando as plantas ainda não tinham definido a última folha (folha bandeira), ou seja, ainda permaneciam na fase vegetativa. Por outro lado, as plantas dos tratamentos com maior vernalização [36 DV $\left(10^{\circ} \mathrm{C}\right.$ durante 6 semanas); $41 \mathrm{DV}$ $\left(4{ }^{\circ} \mathrm{C}\right.$ durante 6 semanas); $46 \mathrm{DV}\left(10{ }^{\circ} \mathrm{C}\right.$ durante 8 semanas) e $54 \mathrm{DV}$ ( $4{ }^{\circ} \mathrm{C}$ durante 8 semanas)] tiveram 39 a 42 folhas/planta e floresceram até o fim do experimento, ou seja, a última folha (folha bandeira) estava presente. 

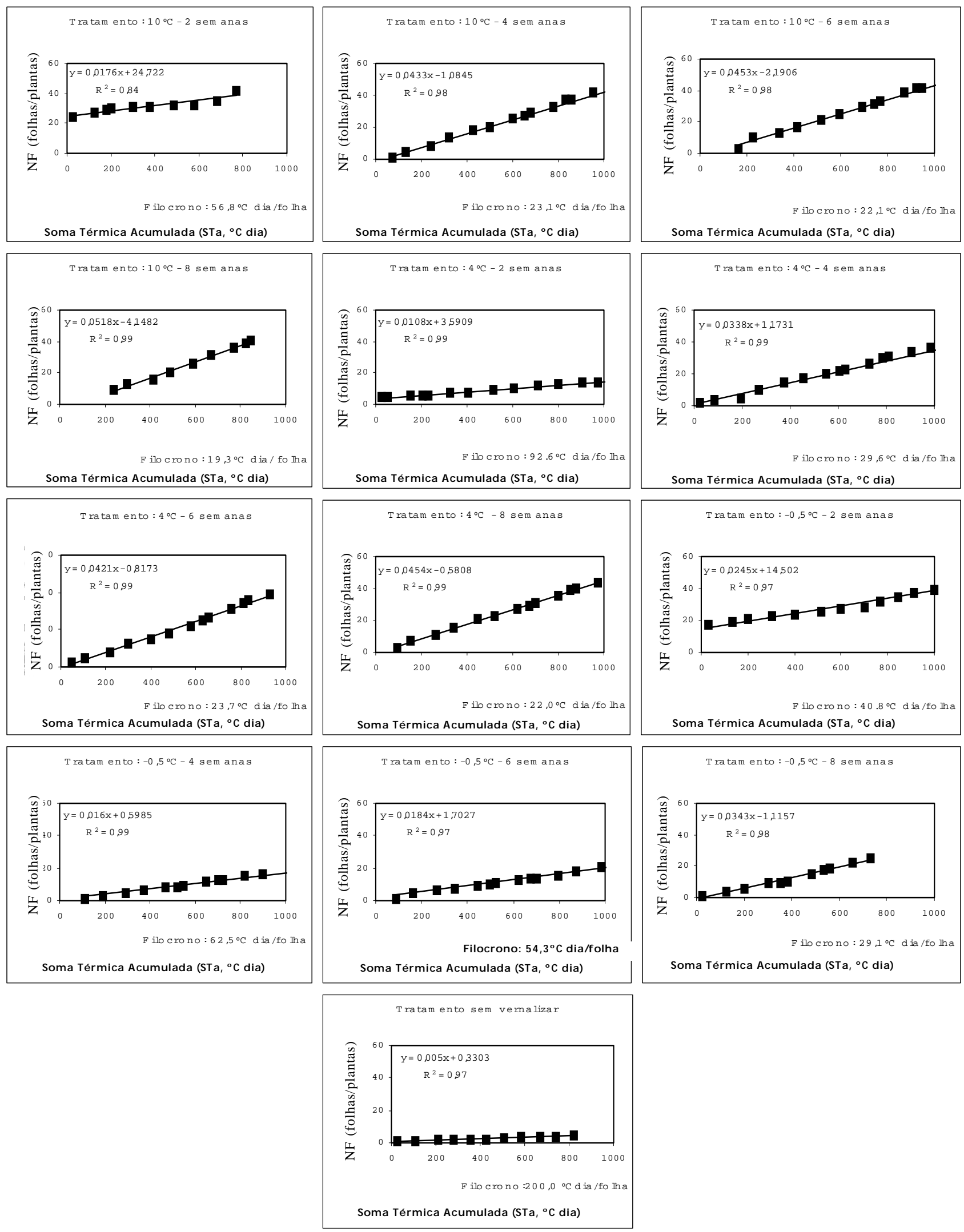

Figura 2. Relação entre número de folhas acumuladas na haste (NF) e a soma térmica acumulada (STa) usada para a estimativa do filocrono em lírio (Lilium longiflorum Thunb.) cv. Snow Queen. Santa Maria (RS), 2003. 


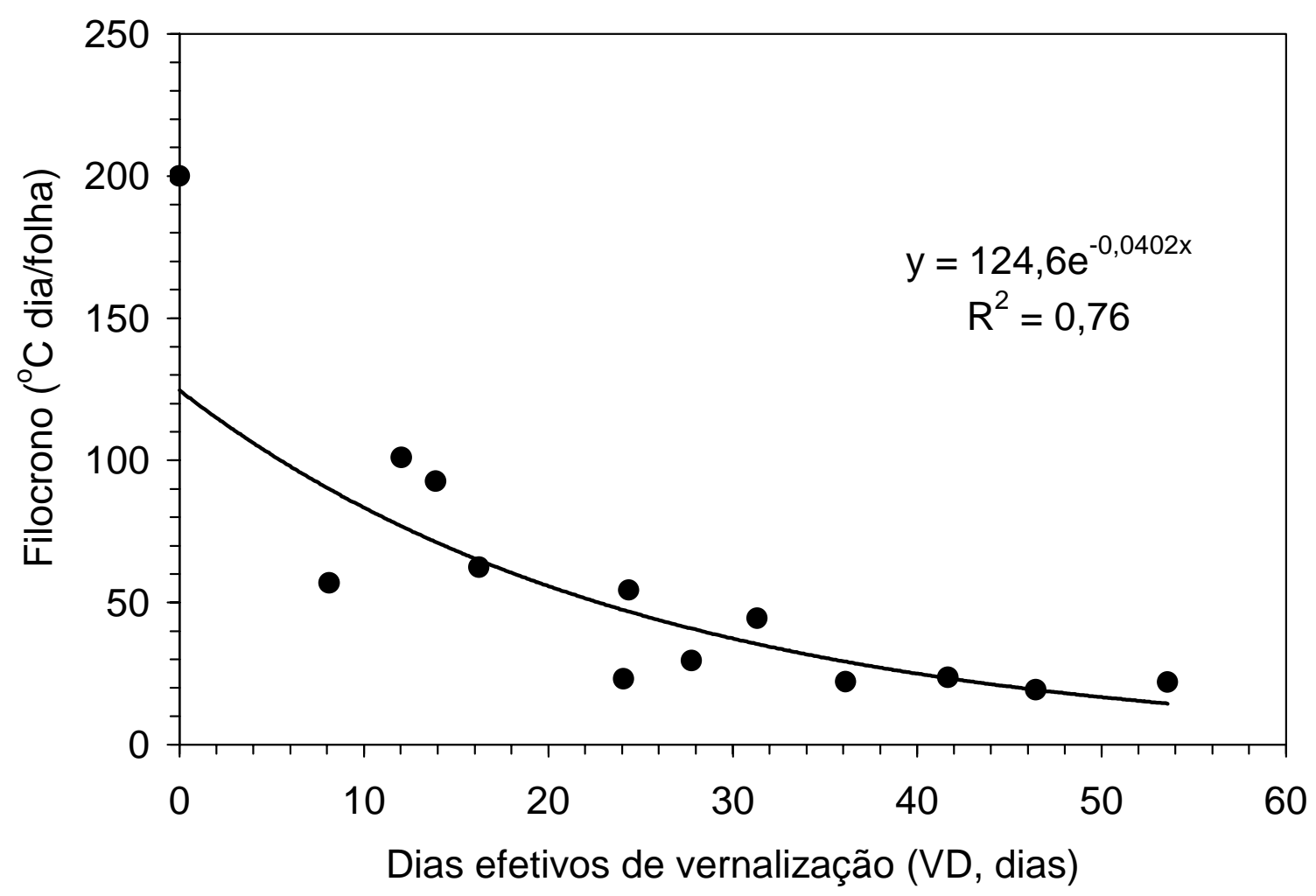

Figura 3. Filocrono de lírio (Lilium longiflorum Thunb.) cv. Snow Queen em função do número de dias efetivos de vernalização. Santa Maria (RS), 2003.

Pelos resultados do presente estudo, portanto, verifica-se que o encurtamento do ciclo da planta de lírio Snow Queen provocado pela vernalização está associado ao efeito combinado da diminuição do número de folhas/planta e do aumento da taxa de aparecimento de folhas (menor filocrono) causados pelo tratamento de frio. Esses resultados com lírio são similares aos obtidos por CUTFORTH et al. (1992), CHUN (1993) e RAwson et al. (1998) que atribuíram o encurtamento do ciclo de desenvolvimento do trigo à diminuição do número total de folhas/planta e à menor taxa de aparecimento de folhas (menor filocrono) pela vernalização. Portanto, um modelo de simulação do efeito da vernalização sobre o desenvolvimento do lírio que seja realístico do ponto de vista biológico deve contemplar a diminuição do número de folhas/planta e o aumento da taxa de aparecimento de folhas (diminuição do filocrono) com o aumento do estado de vernalização das plantas (DV).

\section{CONCLUSÃO}

O valor do filocrono em lírio Snow Queen é afetado pelo seu estado de vernalização. Plantas não vernalizadas ou com pouca vernalização tem um filocrono mais elevado do que plantas completamente vernalizadas. Quando o tratamento de vernalização é maior que 30 dias efetivos de vernalização, o valor do filocrono não aumenta ficando em torno de $25{ }^{\circ} \mathrm{C}$ dia/folha, valor que pode ser utilizado como referência quando os bulbos são vernalizados e utilizados em plantios comerciais.

\section{REFERÊNCIAS}

CAO, W.; MOSS, D.N. Vernalization and phyllochron in winter wheat. Agronomy Journal, Madison, v.83, n.2, p.178-179, 1991.

CHUN, J.U. Variations in rate of leaf emergence, initiation of ear primordium, stem elongation and heading time as affected by vernalization duration of barley with differing growth habits. Field Crops Research, Amsterdam, v.32, n.2, p.159172, 1993.

CUTFORTH, H.W.; JAME, Y.W.; JEFFERSON, P.G. Effect of temperature, vernalization and water stress on phyllochron and final main-stem leaf number of HY320 and Neepawa spring wheats. Canadian Journal of Plant Science, Ottawa, v.72, n.10, p.1141-1151, 1992. 
FISHER, P.R.; HEINS, R.D.; EHLER, N.; LIETH, L.H. A decisionsupport system for real-time management of Easter lily (Lilium longiflorum Thunb.) scheduling and height - I. System description. Agricultural Systems, London, v. 54, n. 1, p. 2337, 1997a.

FISHER, P.R.; HEINS, R.D.; EHLER, N.; LIETH, L.H.; BROGAARD, M.; KARLSEN, P. A decision-support system for real-time management of Easter lily (Lilium longiflorum Thunb.) scheduling and height - II. Validation. Agricultural Systems, London, v.54, n. 1, p. 39-55, 1997b.

KARLSSON, M.G.; HEINS, R.D.; ERWIN, J.E. Quantifying temperature-controlled leaf unfolding rates in "Nellie White" Easter lily. Journal of the American Society of Horticultural Science, Boston, v.113, n.1, p.70-74, 1988.

KLEPPER, B.; RICKMAN, R.W.; PETERSON, C.M. Quantitative characterization of vegetative development in small cereal grains. Agronomy Journal, Madison, v.74, p. 789-792, 1982.

LIETH, J.H.; CARPENTER, P. Modeling stem elongation and leaf unfolding of Easter lily during greenhouse forcing. Scientia Horticulturae, Amsterdam, v.44, p.149-162, 1990.

LE NARD, M. Bases biologicas para la producción y utilización de bulbos en ornamentales. Revista Chapingo, Chapingo, n.1, p.56-59, 1994.

LEE, S.J.; ROH, M.S. Influence of frozen storage duration and forcing temperature on flowering of oriental hybrid lilies. HortScience, Mt. Vernon ,v.36, n.6, p. 1053-1056, 2001.

McRAE, E.A. Lilies: a guide for growers and collectors. Portland: Timber, 1998. 392p.

MOSAAD, M.; ORTIZ-FERRARA, G.; MAHALAKSHMI, V.; FISHER, R.A. Phyllochron response to vernalization and photoperiod in spring wheat. Crop Science, Madison, v.35, n.2, p.168-171, 1995.

MOTA, F. S. Meteorologia Agrícola. 3.ed. São Paulo: Nobel, 1977. 376p.

RAWSON,H.M.;ZAJAC, M.; PENROSE, L.D.J. Effect of seedling temperature and its duration on development of wheat cultivars differing in vernalization response. Field Crops Research, Amsterdam, v.57, n.3, p.289-300, 1998.
ROH, M.S. Flowering response of mid-century hybrid lilies to bulb vernalization and shoot photoperiod treatment. HortScience, Mt. Vernon, v. 20, n.4, p. 710-713, 1985.

ROH, M S.; WILKINS, H.F. The effects of bulb vernalization and shoot photoperiod treatments on growth and flowering of Lilium longiflorum Thunb. cv. Nellie White. Journal of the Americam Society for Horticultural Science, Boston, v.102, n.3, p.229-235,1977a.

ROH, M S.; WILKINS, H.F. Comparison of continuous and alternating bulb temperature treatments on the growth and flowering in Lilium longiflorum Thunb. Journal of the American Society for Horticultural Science, Boston, v.102, n.3, p.242$247,1977 b$.

ROH, M S.; WILKINS, H.F. The control of flowering in Lilium longiflorum Thunb. cv. Nellie White by cyclic or continuous light treatments. Journal of the American Society for Horticultural Science, Boston, v.102, n.3, p.247-253, 1977c.

STRECK, N.A. A generalized nonlinear air temperature response function for node appearance rate in muskmelon (Cucumis melo L.). Revista Brasileira de Agrometeorologia, Santa Maria, v.10, n.1, p.105-111, 2002a.

STRECK, N.A. A generalized vernalization response function for lily (Lilium spp.). Revista Brasileira de Agrometeorologia, Santa Maria, v.10, n.2, p.221-228, 2002b.

STRECK, N.A.; WEISS, A.; BAENZIGER, P.S. A generalized vernalization response function for winter wheat. Agronomy Journal, Madison, v.95, n.1, p.155-159, 2003a.

STRECK, N.A.; WEISS, A.; XUE, Q.; BAENZIGER, P.S. Incorporating a chronology response into the prediction of leaf appearance rate in winter wheat. Annals of Botany, London, v.92, n.2, p.181-190, 2003b.

STRECK, N.A.; WEISS, A.; XUE, Q.; BAENZIGER, P.S. Improving predictions of developmental stages in winter wheat. Agricultural and Forest Meteorology, Amsterdam, v.115, n.3-4, p.139-150, 2003c.

WILHELM, W.W.; MACMASTER, G.S. Importance of the phyllochron in studying development and growth in grasses. Crop Science, Madison, v. 35, n. 1, p.1-3, 1995. 